\title{
Effects of Bradykinin on Aortic Endothelial Function in ApoE-Knockout Mice With Chronic Chlamydia Pneumoniae Infection
}

\author{
Petru Liuba, MD, PhD; Päivi Karnani, MSc ${ }^{\dagger}$; Erkki Pesonen, MD, PhD; Ilari Paakkari, MD, $\mathrm{PhD}^{\dagger}$; \\ Kenneth Persson, MD, PhD*; Anders Forslid, DVM, PhD**
}

\begin{abstract}
Background Impaired muscarinic receptor-mediated vasodilation is an important feature of early atherosclerosis. Earlier studies on apolipoprotein E-knockout mice (apoE-KO) mice suggested adverse effects of Chlamydia pneumoniae infection on the endothelial vasomotor responses of aortas to the muscarinic agonist methacholine. Using additional aorta samples the present study investigated the responses to bradykinin.

Methods and Results ApoE-KO mice were repeatedly inoculated with either Chlamydia pneumoniae (C. pneumoniae) or saline. At 2, 6, and 10 weeks after the first inoculation, precontracted aorta rings from both groups were exposed to bradykinin in the absence and presence of L-NAME and diclofenac. In noninfected animals, the vasomotor responses to bradykinin were similar at all timepoints $(\mathrm{p}>0.5)$. Compared with noninfected animals, the responses in infected animals tended to increase through the study period ( $\mathrm{p}<0.05$ at 10 weeks). Although diclofenac and L-NAME had no effect in noninfected mice, they inhibited the responses to bradykinin in infected mice at 6 and, more markedly, 10 weeks ( $<<0.05$ for both).

Conclusion Bradykinin stimulation of aorta endothelium from C. pneumoniae-infected apoE-KO animals appears to activate compensatory kinin receptor-related mechanisms that could involve nitric oxide and vasorelaxing prostanoids. Although the precise molecular mechanisms require further investigation, one could speculate that strategies increasing bradykinin availability might reverse the arterial dysfunction during chronic infectious disease. (Circ J 2007; 71: 1480-1484)
\end{abstract}

Key Words: Bradykinin; Endothelium; Infection; Relaxation

$\mathbf{T}$ here is evidence suggesting that infection could contribute to the development of atherosclerosis ${ }^{1-3}$ Of infectious pathogens, Chlamydia pneumoniae (C. pneumoniae) has been most often associated with atherosclerosis and its clinical complications.-6 Damage to the vascular endothelium has been hypothesised to underlie in part the putative link between infection and atherosclerosis $^{7-9}$ We have earlier demonstrated that infection of apolipoprotein E-knockout mice (apoE-KO) with C. pneumoniae leads to arterial endothelial dysfunction, as illustrated by impaired endothelium-dependent relaxation of aortas in response to the muscarinic agonist methacholine ${ }^{10}$ The impaired vasomotor response to muscarinic agonists is generally regarded as a hallmark of early atherosclerosis.

A particular phenotype of vascular endothelial dysfunction has been detected in humans with cardiovascular risk factors and without signs of advanced atherosclerosis, being characterized by an impaired muscarinic-mediated vasodilator response associated with normal ${ }^{11-13}$ or even enhanced ${ }^{14}$ responses to bradykinin, another endothelium-dependent vasodilator. Although the mechanisms of this discrepancy

(Received August 17, 2006; revised manuscript received May 1, 2007; accepted May 24, 2007)

Departments of Pediatric Cardiology, *Medical Microbiology, **Laboratory Animal Science, Lund University Hospital and University of Lund, Lund, Sweden and Department of Pharmacology and Toxicology, University of Helsinki, Helsinki, Finland

Mailing address: Petru Liuba, MD, PhD, Division of Pediatric Cardiology, Lund University Hospital, 22185 Lund, Sweden. E-mail: Petru.Liuba@med.lu.se in the vasomotor responses to muscarinic and kinin receptor agonists remain elusive, this observation is in keeping with the hypothesis that the kinin pathway could be used as a pharmacological target in order to improve the endothelial function, and, thereby, to retard the development of atherosclerosis! 15,16

To date, there are no data connecting the endothelial kinin pathway with chronic C. pneumoniae infection, so we investigated the bradykinin-induced vasomotor responses in relation to C. pneumoniae infection in apoE-KO mice. Additional rings from thoracic aorta segments that had been tested with methacholine ${ }^{10}$ were exposed to bradykinin in the present study. The apoE-KO mouse develops atherosclerosis in a similar fashion to humans ${ }^{17}$ and has, therefore, been increasingly used in the past decade for studying the pathogenesis of atherosclerosis in relation to several pathological conditions, including infection! ${ }^{18-20}$

\section{Methods}

\section{Material and Study Design}

Forty-eight apoE-KO mice were purchased from Jackson Laboratories, and fed a regular mouse chow containing $4.5 \%$ crude fat. At the age of 8 weeks, half of the mice were inoculated intranasally with either C. pneumoniae (strain IOL-207; 400,000 infection forming units/mouse/inoculation) or saline. Additional 3 inoculations were carried out at 10,12 , and 14 weeks of age. Prior to inoculation, the mice were sedated with Avertin $(0.1 \mathrm{ml} / 10 \mathrm{~g})$.

Four to eight mice from both infected and noninfected 

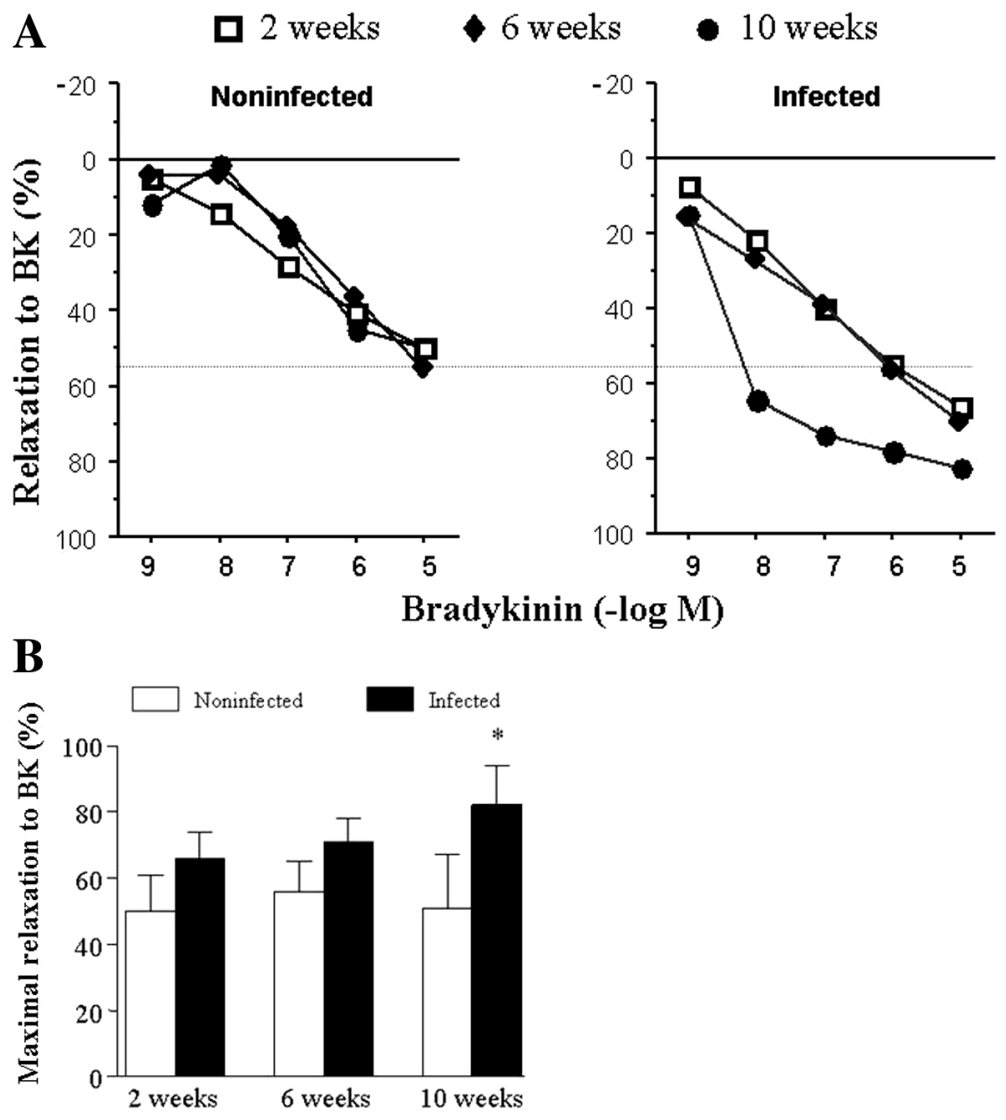

Fig 1. Relaxation curves (A) and maximal relaxations (B) of aortas from noninfected and Chlamydia pneumoniae-infected apolipoprotein E-knockout mice (apoE-KO) mice in response to bradykinin at 2 weeks, 6 weeks, and 10 weeks, respectively. Data are expressed as mean \pm SEM $(n=4-8) ;{ }^{*}<0.05$ vs noninfected.

groups were killed at 2, 6 and 10 weeks after the first inoculation. Prior to this, mice were anaesthetized with sodium pentobarbital $(0.2 \mathrm{mg} / \mathrm{g}$ body weight, ip). This study was conducted in accordance to the local ethics committee guidelines for animal research.

\section{In Vitro Study of Vascular Relaxation Response to Bradykinin}

The thoracic aorta was carefully removed, and placed immediately in fresh Krebs buffer. The vessel was then carefully cleaned of adjacent fatty tissue and cut into 3-mm rings. The aortic rings were suspended by 2 stainless steel hooks in organ chambers containing Krebs solution at $37^{\circ} \mathrm{C}$, bubbled with a mixture of $95 \% \mathrm{O}_{2}$ and $5 \% \mathrm{CO}_{2}$. The arterial smooth muscle tone was measured with a force-displacement transducer connected to a Grass polygraph (Model 7D, Grass Instrument Co, Quincy, MA, USA). The aortic rings were exposed to noradrenaline $(0.1 \mathrm{~mol} / \mathrm{L})$ in order to obtain a $70 \%$ submaximal contraction, and, after reaching a plateau level, relaxed by cumulative addition of sodium nitroprusside (SNP) and bradykinin $\left(10^{-9}\right.$ to $\left.10^{-5} \mathrm{~mol} / \mathrm{L}\right)$ in the absence and presence of $100 \mu \mathrm{mol} / \mathrm{L} \mathrm{NG}_{\text {-nitro-L-arginine }}$ methyl ester (L-NAME, an inhibitor of NO synthesis) or $10 \mu \mathrm{mol} / \mathrm{L}$ diclofenac (an inhibitor of prostaglandin synthesis). These drugs were dropped into the organ bath $30 \mathrm{~min}$ before the administration of noradrenaline.

\section{Drugs}

All drugs were obtained from Sigma Chemical Co ( $\mathrm{St}$ Louis, MO, USA). All concentrations refer to final concentrations in the organ bath.

\section{Statistical Analyses}

The relaxation responses to bradykinin are expressed as percent changes from the pre-contraction levels. Differences between the infected and noninfected mice were calculated by 2-way ANOVA for repeated measures followed by Newman-Keuls test. Statistical significance was accepted at $\mathrm{p}<0.05$ level. All data are expressed as mean \pm SEM; $n$ indicates the number of animals.

\section{Results}

Aortic Relaxation Responses to Bradykinin and SNP

In both infected and noninfected animals, bradykinin caused relaxation of the aorta rings in a concentrationdependent fashion (Fig 1A). For the sake of clarity, the standard errors for maximal relaxation responses are illustrated in Fig 1B. As expected, and conforming to the methacholine-induced relaxation responses observed in our previous studies, ${ }^{10}$ there was no difference in the responses of aortas from noninfected mice at 2, 6, and 10 weeks $(50 \pm$ $11 \%, 56 \pm 9 \%$, and $51 \pm 16 \%$, respectively). As compared with the responses from noninfected animals, the maximal relaxation responses of aortas from infected animals showed a paradoxical trend toward an increase from 2 to 10 weeks, with a significant difference at 10 weeks $(82 \pm 12 \%, \mathrm{p}<0.05$, Fig 1B).

Similarly, the threshold-relaxing concentration $\left(10^{-8} \mathrm{~mol} / \mathrm{L}\right)$ of bradykinin, which had only minimal or no effect in noninfected animals (Fig 1A, Left panel), slightly relaxed the aortas from infected mice at 2 and 6 weeks $(22 \pm 6 \%$ and $26 \pm 8 \%$, respectively), and caused a significant increase in the relaxation response in the infected animals at 10 weeks (65 $\pm 13 \%, \mathrm{p}<0.05$, Figs $1 \mathrm{~A}$ (Right panel), 2B). 
$\nabla$ Diclofenac 6 weeks

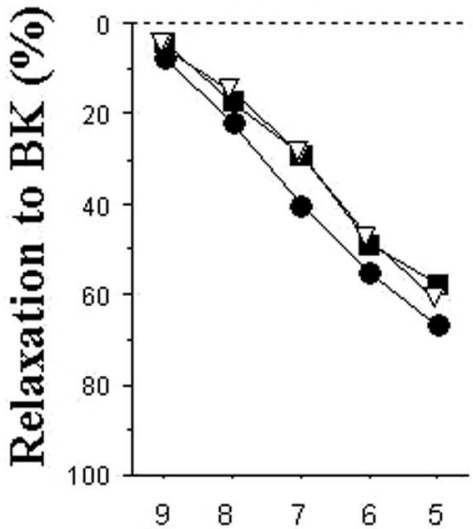

B

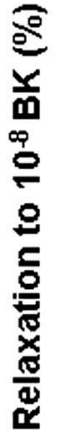

\section{2 weeks}

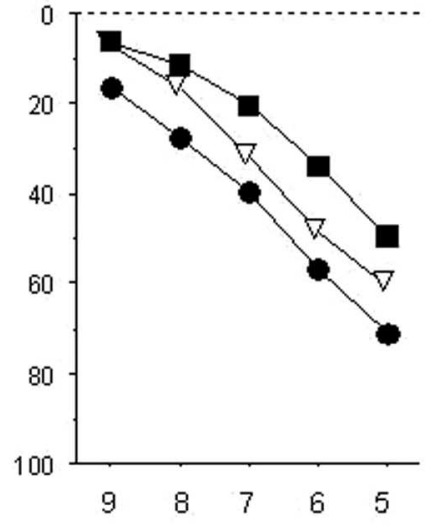

\section{Bradykinin (-log M)}

6 weeks

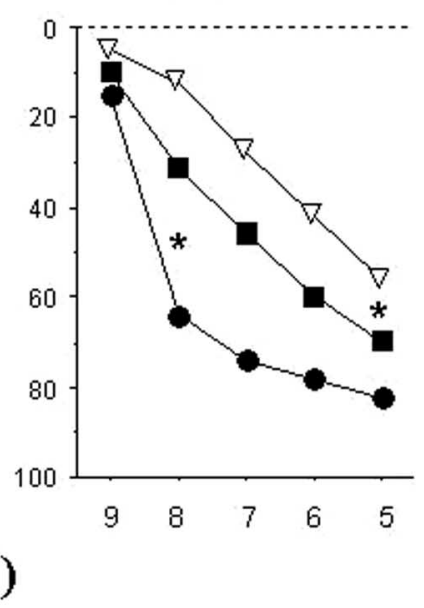

\section{L-NAME}

\section{0 weeks}

10 weeks

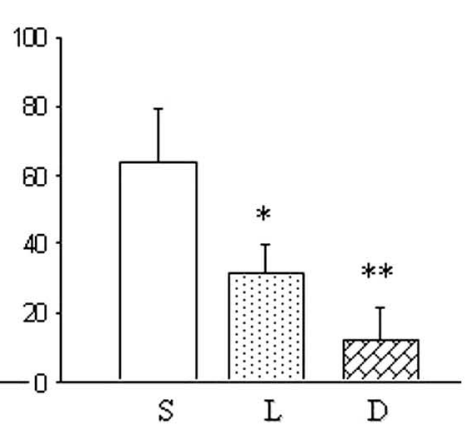

Fig 2. Effects of inhibition of prostaglandin and nitric oxide (NO) synthesis on the relaxation response to bradykinin in Chlamydia pneumoniae-infected apolipoprotein E-knockout mice (apoE-KO) mice at 2 weeks, 6 weeks, and 10: (A) cumulative addition and (B) $10^{-8} \mathrm{~mol} / \mathrm{L}$. Data obtained without pretreatment (solvent, S), and in the presence of L-NAME

(L) or diclofenac (D). Data are expressed as mean \pm SEM $(n=5-8) ; * p<0.05$, and $* * p<0.01$ vs solvent at 10 weeks.

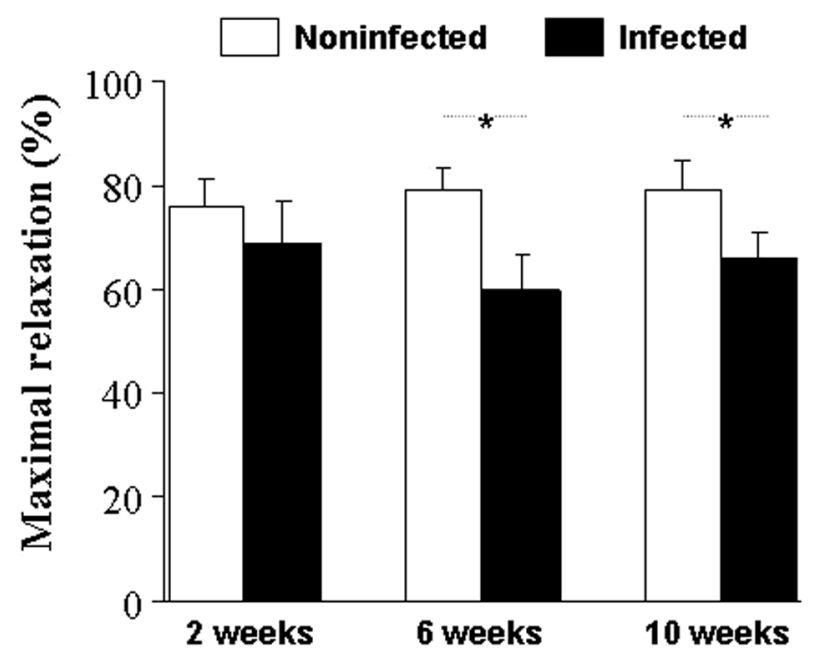

Fig 3. Maximal relaxation responses to methacholine at 2, 6 and 10 weeks in noninfected and Chlamydia pneumoniae-infected apolipoprotein E-knockout mice. Data are expressed as mean \pm SEM $(n=5-8)$; $* \mathrm{p}<0.05$.
The relaxation responses to SNP were similar in both groups at each time point ( 2 weeks: $\mathrm{p}=0.5 ; 6$ weeks: $\mathrm{p}=0.4$; 10 weeks: $\mathrm{p}=0.5$ ).

\section{Effects of L-NAME and Diclofenac on Bradykinin-Induced} Relaxation

Inhibition of either NO synthesis by L-NAME or prostaglandin synthesis by diclofenac, or the combination of these 2 had no significant effect on the bradykinin-induced relaxation of aortas from noninfected animals (data not shown). The relaxation responses to bradykinin were not significantly modified by these 2 inhibitors in infected animals at 2 and 6 weeks (Figs 2A,B). Similarly, no significant effect was obtained with combined pre-treatment (data not shown). However, in infected mice at 10 weeks, L-NAME and diclofenac decreased the relaxation to the thresholdrelaxing concentration $\left(10^{-8} \mathrm{~mol} / \mathrm{L}\right)$ by approximately $30 \%$ $(\mathrm{p}<0.05)$ and $50 \%(\mathrm{p}<0.01)$, respectively $($ Fig $2 \mathrm{~B})$. The maximal responses were also inhibited by these drugs, with a significant difference for diclofenac $(56 \pm 10 \%, \mathrm{p}<0.05)$. At this time-point, when used together, L-NAME and diclofenac had a slightly greater, yet statistically nonsignificant inhibitory effect compared with that caused by diclofenac alone $(\mathrm{p}=0.2)$. 
Effects of B2-Receptor Antagonist Hoe-140 on

Bradykinin-Induced Relaxation

Pretreatment of aortas with Hoe-140 abolished the responses to bradykinin in all animals at each time-point (mean value of maximal relaxation: $0-10 \%$ ) with no significant difference between the groups $(\mathrm{p}>0.5$ at each timepoint).

\section{Discussion}

Previous studies from our laboratory ${ }^{10}$ have shown that chronic C. pneumoniae infection of apoE-KO mice may cause injury to the aortic endothelium, as indicated by the impaired aortic relaxation responses to the muscarinic agonist methacholine (Fig 3). In the same animal model, infection with C. pneumoniae caused lipid accumulation and inflammatory changes in the aorta wall ${ }^{9}$

Using additional segments from the aortas previously tested with methacholine, the findings of the present study indicate that the responses to bradykinin are not altered in the early phase of chronic infection with C. pneumoniae. Moreover, the augmented relaxation responses to bradykinin in the later stages of infection suggest that active regulatory mechanisms in response to bradykinin stimulation could be responsible for the observed differences. Although pretreatment with the bradykinin B2-receptor antagonist Hoe140 abolished the relaxation response to bradykinin in infected animals, the contribution of vascular endothelial kinin B1 receptors, which may be upregulated by infections and inflammation, ${ }^{21}$ might be among the underlying mechanisms.

Earlier studies on vascular physiology in relation to various risk factors for atherosclerosis have suggested a discrepancy between the vasomotor responses to muscarinic agonists and those elicited by bradykinin. Indeed, in the majority of studies, impaired arterial vasomotor responses to muscarinic receptor stimulation were documented, whereas the responses to bradykinin were shown to be preserved $^{11-13}$ or even enhanced ${ }^{14}$ It has therefore been speculated that the endothelial injury in atherosclerosis is receptor-specific, being confined at least in the early stages to muscarinic receptors. The mechanisms proposed to explain these findings have primarily depicted 2 possible scenarios: either the kinin receptor pathway is spared in the early stages, or regulatory kinin-mediated, endotheliumdependent mechanisms are set in response to the injurious stimuli. Such mechanisms might involve kinin-mediated upregulation of endothelial pathways such as increased synthesis of cyclooxygenase (COX)-dependent relaxant prostaglandins? ${ }^{2}$

Although the arterial relaxation responses to methacholine are largely mediated by NO, the relaxation to bradykinin might in part involve both $\mathrm{NO}$ and prostacyclin, a COX product with dilatory properties ${ }^{16}$ However, in the present study, blocking of either NO synthesis or COX activity by L-NAME and diclofenac, respectively, did not have a consistent inhibitory effect on the bradykinininduced relaxation in the noninfected apoE-KO mice. This suggests that at least in this animal model a non-NO, nonprostanoid factor, possibly endothelial-derived hyperpolarizing factor, is mainly responsible for the relaxant effects of bradykinin. In contrast, pretreatment of aortas from infected animals with L-NAME or diclofenac inhibited the relaxation responses to bradykinin, suggesting that increased production of both $\mathrm{NO}$ and relaxing prostaglandins, most likely prostacyclin, are responsible for the augmented relaxation to bradykinin in the infected animals. Because the amounts of the COX and NOS enzyme proteins did not differ between infected and noninfected animals ${ }^{10}$ it is possible that the activity of both the NOS and COX pathways could have been upregulated by bradykinin in infected animals, with a composite result of increased availability of $\mathrm{NO}$ and relaxing prostaglandins.

Bradykinin could improve the bioactivity of NO by serving as a substrate to NOS because of the L-arginine incorporated in its structure ${ }^{23}$ and by its interaction with tetrahydrobiopterin ${ }^{24}$ the coenzyme implicated in the NO synthesis. This results in a better coupling of L-arginine and NADPH oxidation, leading to improved bioactivity of NO. These bradykinin-induced mechanisms may be important in the setting of vascular pathologies, including atherosclerosis, in which the levels of both L-arginine and tetrahydropterin are suboptimal25,26 Of note, L-arginine was shown to improve the coronary reactivity in swine with $\mathrm{C}$. pneumoniae infection following repeated inoculations with this pathogen ${ }^{27}$ a finding that supports the hypothesis that decreased availability of endothelial L-arginine could be associated with this infection. The improvement in the NOS pathway by bradykinin could in turn influence the COX pathway, probably in part through a complex cross-talk between the NOS and COX enzymes, ${ }^{28}$ with a subsequent increase in the release of relaxant prostaglandin.

Under normal circumstances, bradykinin contributes to regulation of both the resting tone and flow-mediated dilatation of arteries, and exerts important antiatherogenic effects through $\mathrm{NO}$ and prostacyclin?29,30 Of note, angiotensin-converting enzyme (ACE) inhibitors improve vascular endothelial function in various pathological conditions associated with atherosclerosis, in part through increased availability of bradykinin? 31,32 Accumulating data from both experimental studies and clinical trials suggest that ACE inhibitors may retard the development of atherosclerosis and its manifestations ! $^{18,33,34}$ Based on the present findings, one might speculate that similar benefit could be attained by ACE inhibitors on C. pneumoniae-induced endothelial dysfunction and, perhaps, on the development of atherosclerosis with an infectious basis.

In conclusion, bradykinin stimulation of the endothelium of aortas from C. pneumoniae-infected apoE-KO animals appears to activate compensatory kinin receptor-related mechanisms that could involve NO and vasorelaxing prostanoids. Additional studies are needed to further investigate the molecular pathways, and, in particular, to verify whether similar effects may occur in other animal models.

\section{Acknowledgments}

This work was supported by grants from the Lund University and the Swedish Medical Association. We thank Mrs Anneli von Behr and Mrs Nada Bechtali for their technical assistance.

\section{References}

1. Libby P, Egan D, Skarlatos S. Role of infectious agents in atherosclerosis and restenosis: An assessment of the evidence and need for future research. Circulation 1997; 96: 4095-4103.

2. Leinonen M, Saikku P. Evidence for infectious agents in cardiovascular disease and atherosclerosis. Lancet 2002; 2: 11-17.

3. Epstein SE. The multiple mechanisms by which infection may contribute to atherosclerosis development and course. Circ Res 2002; 90: $2-4$.

4. Jitsuiki K, Yamane K, Nakajima M, Nakanishi S, Tasaki N, Watanabe H, et al. Association of Chlamydia pneumoniae infection and carotid intima-media wall thickness in Japanese Americans. Circ J 2006; 70: 815-819. 
5. Ngeh J, Anand V, Gupta S. Chlamydia pneumoniae and atherosclerosis: What we know and what we don't. Clin Microbiol Infect 2002; 8: $2-13$.

6. Saikku P. Seroepidemiology in Chlamydia pneumoniae-atherosclerosis association. Eur Heart J 2002; 23: 263-264.

7. Gimbrone M, Bevilacqua M, Cybulski M. Endothelial-dependent mechanisms of leukocyte adhesion in inflammation and atherosclerosis. Ann NY Acad Sci 1990; 598: 77-85.

8. Mayr M, Metzler B, Kiechl S, Willeit J, Schett G, Xu Q, et al. Endothelial cytotoxicity mediated by serum antibodies to heat shock proteins of Escherichia coli and Chlamydia pneumoniae: Immune reactions to heat shock proteins as a possible link between infection and atherosclerosis. Circulation 1999; 99: 1560-1566.

9. Coombes BK, Chiu B, Fong IW, Mahony JB. Chlamydia pneumoniae infection of endothelial cells induces transcriptional activation of platelet-derived growth factor-B: A potential link to intimal thickening in a rabbit model of atherosclerosis. J Infect Dis 2002; 185: $1621-1630$.

10. Liuba P, Karnani P, Pesonen E, Paakkari I, Forslid A, Johansson L, et al. Endothelial dysfunction after repeated Chlamydia pneumoniae infection in apolipoproteinE-knockout mice. Circulation 2000; 102: $1039-1044$.

11. Prasad A, Hussain S, Schenke W, Mincemoyer R, Epstein N, Quyyumi AA. Contribution of bradykinin receptor dysfunction to abnormal coronary vasomotion in humans. J Am Coll Cardiol 2000; 36: $1467-1473$.

12. Aptecar E, Teiger E, Dupouy P, Benvenuti C, Kern MJ, Woscoboinik $\mathrm{J}$, et al. Effects of bradykinin on coronary blood flow and vasomotion in transplant patients. J Am Coll Cardiol 2000; 35: 1607-1615.

13. Kuga T, Egashira K, Mohri M, Tsutsui H, Harasawa Y, Urabe Y, et al. Bradykinin-induced vasodilation is impaired at the atherosclerotic site but is preserved at the spastic site of human coronary arteries in vivo. Circulation 1995; 92: 183-189.

14. Vajo Z, Szekacs B, Mc Donald M, Takahashi B, Srivathsan K, Dachman WD. Paradoxically enhanced bradykinin-induced venodilation in young, healthy, short-term smokers. J Cardiovasc Pharmacol 1999; 34: 316-319.

15. Hartman J. The role of bradykinin and nitric oxide in the cardioprotective action of ACE inhibitors. Ann Thorac Surg 1995; 60: 789792.

16. Hall JM. Bradykinin receptors: Pharmacological properties and biological roles. Pharmacol Ther 1992; 56: 131-190.

17. Nakashima Y, Plump AS, Raines EW, Breslow JL, Ross R. ApoEdeficient mice develop lesions of all phases of atherosclerosis throughout the arterial tree. Arterioscler Thromb 1994; 14: 133-140.

18. Okabe TA, Kishimoto C, Shimada K, Murayama T, Yokode M, Kita $\mathrm{T}$. Effects of late administration of immunoglobulin on experimental atherosclerosis in apolipoproteinE-deficient mice. Circ J 2005; 69: $1543-1546$

19. Moazed T, Kuo C, Grayston JT, Campbell LA. Murine models of Chlamydia pneumoniae infection and atherosclerosis. J Infect Dis 1997; 175: 883-890

20. Hsich E, Zhou YF, Paigen B, Johnson TM, Burnett MS, Epstein SE.
Cytomegalovirus infection increases development of atherosclerosis in apolipoprotein-E knockout mice. Atherosclerosis 2001; 156: 23-28.

21. Marceau F, Hess J, Bachvarov D. The B1 receptors for kinins. Pharmacol Rev 1998; 50: 357-386.

22. Puybasset L, Béa ML, Ghaleh B, Giudicelli JF, Berdeaux A. Coronary and systemic hemodynamic effects of sustained inhibition of nitric oxide synthesis in conscious dogs: Evidence for cross talk between nitric oxide and cyclooxygenase in coronary vessels. Circ Res 1996; 79: 343-357.

23. Volpe A, Giardina B, Preziosi P, Carmignani M. Biosynthesis of endothelium-derived nitric oxide by bradykinin as endogenous precursor. Immunopharmacology 1996; 33: 287-290.

24. Golser R, Gorren AC, Leber A, Andrew P, Habisch HJ, Werner ER, et al. Interaction of endothelial and neuronal nitric oxide synthases with the bradykinin $\mathrm{B} 2$ receptor: Binding of an inhibitory peptide to the oxygenase domain blocks uncoupled NADPH oxidation. J Biol Chem 2000; 275: 5291-5296.

25. Tiefenbacher CP, Bleeke T, Vahl C, Amann K, Vogt A, Kübler W. Endothelial dysfunction of coronary resistance arteries is improved by tetrahydrobiopterin in atherosclerosis. Circulation 2000; 102: $2172-2179$.

26. Aji W, Ravalli S, Szabolcs M, Jiang XC, Sciacca RR, Michler RE, et al. L-arginine prevents xanthoma development and inhibits atherosclerosis in LDL receptor knockout mice. Circulation 1997; 95: 430-437.

27. Liuba P, Pesonen E, Paakkari I, Forslid A, Persson K. The effects of repeated Chlamydia pneumoniae infection on coronary arteries in piglets: Are these precursive to atherosclerosis? (abstract). $J$ Heart Dis 2001; 2: 33 .

28. Osanai T, Fujita N, Fujiwara N, Nakano T, Takahashi K, Guan W, et al. Cross talk of shear-induced production of prostacyclin and nitric oxide in endothelial cells. Am J Physiol 2000; 278: H233-H238.

29. Cherry PD, Furchgott RF, Zawadski JV, Jothianandan D. Role of endothelial cells in relaxation of isolated arteries by bradykinin. Proc Natl Acad Sci USA 1982; 79: 2106-2110.

30. Henderson AH. Endothelium in control. Br Heart J 1991; 65: 116125.

31. Varin R, Mulder P, Tamion F, Richard V, Henry JP, Lallemand F, et al. Improvement of endothelial function by chronic angiotensinconverting enzyme inhibition in heart failure: Role of nitric oxide, prostanoids, oxidant stress, and bradykinin. Circulation 2000; 102: $351-256$.

32. Zanzinger J, Zheng X, Bassenge E. Endothelium dependent vasomotor responses to endogenous agonists are potentiated following ACE inhibition by a bradykinin dependent mechanism. Cardiovasc Res 1998; 28: 209-214.

33. Halkin A, Keren G. Potential indications for angiotensin-converting enzyme inhibitors in atherosclerotic vascular disease. Am J Med 2002; 112: 126-134.

34. Yusuf S. From the HOPE to the ONTARGET and the TRANSCEND studies: Challenges in improving prognosis. Am J Cardiol 2002; 89: $18 \mathrm{~A}-26 \mathrm{~A}$. 